\title{
KAPITULATION UND \\ GEFANGENNAHME DER RHEINARMEE
}

\author{
„Wer davon lebt, einen Feind zu bekämpfen, hat Interesse \\ daran, daß er am Leben bleibt."
}

Nietzsche ${ }^{1}$

Am 27. Oktober 1870 endete die Belagerung von Metz. Auf deutscher Seite, wo der Glaube daran zum Schluß fast geschwunden war, sprach man jetzt nicht mehr von Kriegskunst, sondern von Gott ${ }^{2}$. Die französische Rheinarmee kapitulierte nahezu bedingungslos, nachdem ihre Unterhändler bis zum Schluß vergeblich versucht hatten, das Schicksal der Armee von dem der Festung zu trennen. Die Präliminarien hatten Prinz Friedrich Karl und der greise General Changarnier ausgehandelt, der schon unter Napoleon I. gedient hatte und jenseits von Kaiserreich oder Republik für die großen soldatischen Traditionen Frankreichs zu stehen schien. Dieser und General Jarras, der dann am Kapitulationstag auf Schloß Frescaty südlich von Metz die französische Feder führte, konnten lediglich noch erwirken, daß den kriegsgefangenen Offizieren gestattet wurde, ihre Degen zu behalten ${ }^{3}$. Das war übrigens mehr als eine Geste, denn ein Offizier ohne seinen Degen galt damals wie später im Frieden als nackt und achtete daher in der Öffentlichkeit streng darauf, seine Waffe nirgends abzulegen. Sie war ein Symbol des militärischen Standes und dessen Unangreifbarkeit ${ }^{4}$. Ein zusätzliches Verlangen, je ein Regiment Infanterie, Kavallerie und eine Batterie Artillerie „mit klingendem Spiel und fliegenden Fahnen" nach Algerien abmarschieren zu lassen, wurde dagegen abgelehnt ${ }^{5}$. Um 11 Uhr abends telegraphierte der Prinz aus Corny an seinen Vater König Wilhelm nach Versailles:

Heute Abend 10 Uhr im Schlosse Capitulation durch General von Stiehle abgeschlossen. Am 29. werden 173000 Mann mit 3 Marschällen und über 6000 Offizieren kriegsgefangen und Forts wie Festung Metz von uns besetzt. ${ }^{6}$

Am Ende hatte der Hunger die Rheinarmee zur Aufgabe gezwungen. Immer in besseren Quartieren und geschützteren Positionen als die Belagerer und zudem ausreichend mit Geschützen und Munition versehen, mußte sie dennoch die Waffen strecken, weil es früher versäumt worden war, den Fall einer defensiven Kriegslage einzukalkulieren und entsprechende Maßregeln zu treffen. Die Vorräte an Brot, das zum Ende überhaupt nur noch durch im

${ }^{1}$ Menschliches, Allzumenschliches, KSA, Bd. 2, S. 326.

2 Vgl. KRETSCHMAN, Kriegsbriefe, S. 187.

3 Vgl. Protokoll der Verhandlung über die Kapitulation im Anhang/Anlage VI.

4 Vgl. FREVERT, Militär, S. $171 \mathrm{f}$.

5 HiRTH, Tagebuch, Bd. II, S. 2945.

6 Telegraphische Kriegsbotschaften, S. 34. 


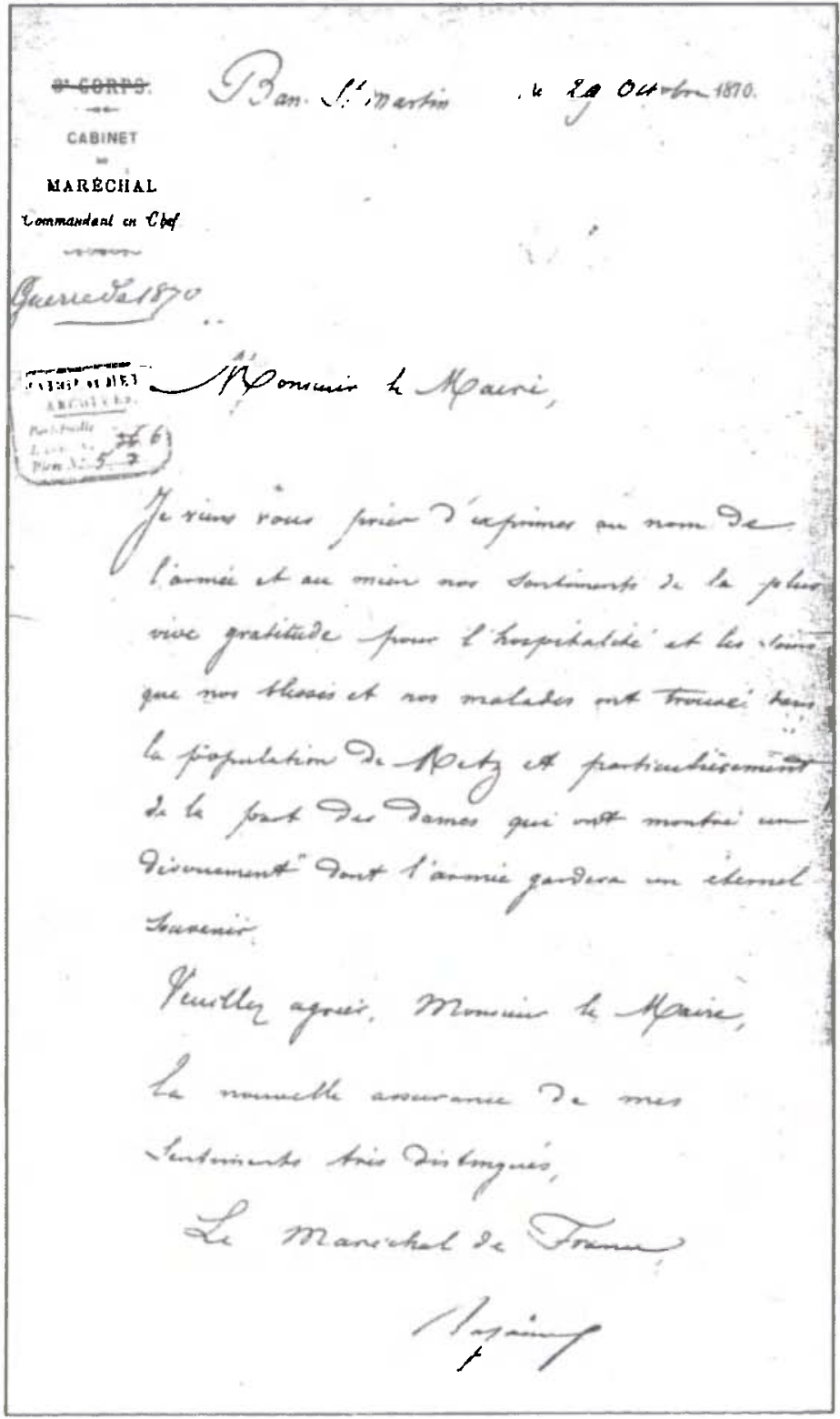

29 octobre 1870. A son depart, le maréchal Bazaine remercie la population messine.

Abb. 14: Quelle: DeNIS, Garnison. 
Grunde ungenießbare Zusätze gebacken werden konnte7, hatte man bereits Tage vor der Kapitulation aufgebraucht, und Pferdefleisch konnten die meisten Soldaten kaum noch riechen. Die Zeiten waren ohnehin vorüber, in denen die Leute ihre Stiefel aßen, ehe sie sich ergaben ${ }^{8}$. Selbst eine im „kriegskommunistischen" Stil durchgeführte Beschlagnahmung sämtlicher vorhandenen Reserven ohne Rücksicht auf Stand und Besitz hätte die Festung wohl kaum erheblich länger in die Lage versetzt, Widerstand zu leisten'. Auch wären derartige Maßnahmen vermutlich an der starken sozialen Ausdifferenzierung der Stadtbevölkerung wie der zuletzt fast explosiven Spannungslage zwischen kaiserlichen Militärs und republikanischer Einwohnerschaft gescheitert und hätten vermutlich in den Bürgerkrieg geführt.

„Vom Hunger besiegt" zaines, „müssen wir das Gesetz des Krieges ertragen und uns gefangen geben." 10 Jedoch meinten viele, daß es bis dahin hätte nie kommen dürfen, und man kreidete Marschall Bazaine nicht nur von französischer Seite an, den befreienden Ausbruch mit letzter Entschlossenheit nicht gewagt zu haben:

Aber heraus hätten sie gemußt, wenn sie wirklich ernstlich gewollt hätten; keine preuBische Armee von derselben Stärke würde sich haben so einschließen lassen, sie hätten uns an vielen Stellen, z. B. da, wo wir zuletzt gestanden ${ }^{11}$, fast ohne Waffen beiseite drücken können; wir haben auf einer gefüllten Pulvertonne gelebt, ohne die Größe der Gefahr zu erkennen, die über uns schwebte. ${ }^{12}$

In der Stadt hatte sich schon vor der Kapitulation, es war bereits die Rede davon, eine brodelnde Stimmung gegen die Führung der Rheinarmee und den kaisertreuen Marschall im besonderen entwickelt. Die Zeilen eines französischen Offiziers, geschrieben nach dem gescheiterten Ausfall vom 7. Oktober, klagten Bazaine der Verantwortung für die hoffnungslose Lage an und brachten gleichsam jene Argumente gegen ihn vor, die nach dem Krieg zu seiner Verurteilung führen sollten ${ }^{13}$ :

Warum haben sie, als sie am 26. August auf einiger einzigen Strasse ihre Armee vor St. Julien konzentriert hatten, unter dem Vorwand, dass das Wetter schlecht sei, keine Schlacht geliefert? Regnete es nicht eben so gut für die Preussen als für sie? Sie wussten augenscheinlich, dass die Armee Mac-Mahons vom Norden her auf dem Marsche sei,

7 HÉrisSON, Legende, S. 195.

8 Ibid S. 136.

9 Stadtkommandant Coffinières hatte Bazaine bereits am 8 . Oktober informiert, daß auch bei vollständiger Beschlagnahmung aller Vorräte kaum länger als bis zum 20 . Oktober Zeit blieb. Ibid S. 135.

10 FIRCKS, Vertheidigung, S. 465.

11 Bei Woippy nordwestlich von Metz.

12 RindfleisCH, Feldbriefe, S. 89.

13 Bazaine wurde 1873 von einem Kriegsgericht zum Tode verurteilt und später zu lebenslanger Verbannung begnadigt, weil er kapituliert hatte, "ohne seine Verteidigungsmittel zu erschöpfen und vorher alles gethan zu haben, was ihm Pflicht und Ehre vorschrieben. "CHUQUET, Der Krieg, S. 151. Dazu ausführlich: HéRISSON, Legende. 
und ich glaube, dass es ihnen gelungen wäre, ihm die Hand zu reichen [...]. Warum haben sie am 31. August, selbst während der Nacht, die Vortheile nicht verfolgt, welche die Armee errungen, und warum behielten sie nicht die Stellungen, welche dieselbe mit ihrem Blut erkauft [...]. Warum haben sie am 7. Oktober auf der Ebene von Thionville einen großen Kampf geliefert? Was bezweckten sie? Sie wollten sich verproviantieren, so behauptet man [...]; anstatt einige Regimenter in den Kampf zu senden, mussten sie ein Armeecorps, nötigenfalls zwei ins Feuer bringen. Ungeachtet dessen gelang es der Tapferkeit unserer Soldaten, sich in den Besitz von Grandes Tapes ${ }^{14}$ zu setzen, wo sie wohlausgestattete Vorratskammern vorfanden. Aber den Erfolg, sie wollten ihn nicht, weil, nachdem sie denselben um den Preis des Blutes von ungefähr 1000 unserer Soldaten $^{15}$ errungen, der Rückzug befohlen wurde [...]. Schliesslich die ernsteste Frage. Warum haben sie die feindliche Einschliessungsamee nicht jeden Tag, zu jeder Stunde, durch grössere Ausfälle beunruhigt und angegriffen? Dieses wäre ihnen ein Leichtes gewesen, und sie hätten jeden Tag, jede Stunde, wenn auch keine entscheidenden Erfolge erringen, doch der Belagerungsarmee Verluste beibringen können, welche sie erschöpft und demoralisiert hätten [...]. Sie haben nichts gethan [...].16

Zuletzt hatten sich sogenannte Perceurs gefunden - also solche, die unter allen Umständen den Durchbruch wagen wollten. Von der anfangs großen Zahl Ambitionierter blieben jedoch am Abend des 27. Oktobers noch etwa zehn Offiziere und 50 Mann übrig, die von Bellecroix her gegen die deutschen Linien vorgingen, um den Tod oder die Freiheit zu suchen. Die Aktion schlug wegen Sturm und Regen vollkommen fehl, und nur dem Kommandanten Leperche soll es gelungen sein, ins Innere Frankreichs durchzubrechen $^{17}$. Gegen die Heißsporne stand Bazaines ruhiges Kalkül, der seine erste Aufgabe darin sah, die Armee zu schonen und nicht in einer zwecklosen Schlacht oder gar um der Revolution willen, deren Gegner er war, zu riskieren. Auch stellte sich die simple Frage nach der Versorgung der dann zu erwartenden Verwundeten, für die in Metz keinerlei Unterbringungs- und Pflegemöglichkeiten mehr bestanden ${ }^{18}$. Im Ausland erwog man, daß „politische Beweggründe die militärische Aktion gelähmt haben"19. Nach Bekanntwerden der Kapitulation gab es hier und da spontanen Widerstand gegen die bevorstehende Übergabe der Stadt. Eine Reihe junger Offiziere wollten sich nicht „wie eine Herde Hammel“ 20 verkaufen lassen. Es scheint aber, als habe gerade diese gegen die eigene Armeeführung gerichtete Wut letztendlich den

14 Ortschaft nördlich von Metz an der Straße nach Thionville.

15 Nach offiziellen Angaben forderte der sogenannte „combat des Tapes, Bellevue et Saint Remy" vom 7. Oktober französischerseits 1257 Mann an Toten, Verwundeten und Vermißten: BaZAINe, L' Armée, S. $214 \mathrm{f}$.

16 HiRTH, Tagebuch, Bd. II, S. $2227 \mathrm{f}$.

17 Vgl. WestPHAL, Metz, Teil III, S. $323 \mathrm{f}$.

18 HÉRISSON, Legende, S. 133.

19 ENGELS, Der Fall von Metz. In: MEW, Bd. 17, S. 154-57, hier S. 156.

20 HIRTH, Tagebuch, Bd. II, S. 2228. Am Nachmittag des 28. Oktober kam es durch einen Garnisonsoffizier zu einem letzten Versuch, die Nationalgarde zum Widerstand aufzustacheln und die Stadttore vor den Deutschen verschließen zu lassen. Ein junger Mann schwang sich auf ein weißes Pferd, forderte die Menge auf sich zu verteidigen und stimmte die Marseillaise an. Anschließend zog er seinen Revolver und schoß dreimal in die Luft: ConTAMINE, Metz, S. 375. 
Deutschen genützt, denn bei der Übernahme der Stadt kam es zu keinerlei Zwischenfällen.

Mit der Kapitulation von Metz war für die Sieger ein wichtiges Kriegsziel erreicht, was unmittelbare verwaltungstechnische Neuerungen nach sich zog. So wie nach dem Fall von Straßburg der Präfekt des Elsaß’ seinen Sitz von Hagenau sogleich an den Rhein verlegt hatte, siedelte nun jener von Deutsch-Lothringen, Graf Henckel von Donnersmarc, aus Saargemünd ins Metzer Präfekturgebäude über. Noch im August waren die elsässischen Departements Haut-Rhin und Bas-Rhin sowie das lothringische Departement Moselle, einschließlich Metz, und Teile der Departements Vosges und Meurthe zu einer Verwaltungseinheit von etwa 15000 Quadratkilometern unter provisorischer deutscher Administration zusammengefaßt worden, die dem späteren „Reichsland“ entsprach ${ }^{21}$. Die Würfel für ein deutsches Lothringen waren also gefallen, lange vor der offiziellen Annexion. Auch schien der Krieg nun entschieden zu sein. So mutmaßte die englische Times, daß mit dem Fall von Metz der Widerstand Frankreichs gebrochen sei: „Metz“, so hieß es da, "war viel stärker als Paris, und Metz ist gefallen."22 Während sich die französische Republik von den "kaiserlichen Versagern“ um Bazaine abwandte, würdigten die Preußen ihren Erfolg auch äußerlich: König Wilhelm ernannte den Kronprinzen und seinen Neffen, Prinz Friedrich Karl, zu Feldmarschällen und erhob General Moltke nach Vollendung seines 70 . Geburtstages in den Grafenstand. Für die unteren Chargen fielen jede Menge Eiserner Kreuze ab.

Am 29. und 30. des Monats zogen die französischen Truppen korpsweise aus der Stadt. Es goß in Strömen, als Zehntausende demoralisierter Soldaten unter den Augen der Gewehr bei Fuß stehenden Deutschen sternförmig aus der Festung marschierten. Viele schleppten sich krank und entkräftet auf den verschlammten Straßen dahin. Disziplin und Haltung einzelner Truppenteile waren heruntergekommen, und „ein Teil der Mannschaften war betrunken"23. Das Gros der Rheinarmee jedoch, besonders das Gardekorps und das Korps Canrobert, machte immer noch einen achtungsgebietenden Eindruck, und die anerkennenden Stimmen überwogen bei weitem:

Der Vorbeimarsch fand mit einer Ruhe und Ordnung statt, die die vortreffliche Disziplin der Truppen zeigte, die sich noch in einer so kritischen und schmerzlichen Lage erkennen ließ. Fast alle Truppen bestanden aus sehr schönen Leuten, sie waren gut bekleidet und gingen in guter Haltung still vorbei. Vielen alten Soldaten rannen die Tränen aus den Augen. Bei dem ganzen Korps Canrobert habe ich nur drei Leute gesehen, die betrunken waren; bei einer Truppenzahl von nahezu 30.000 Mann, die genötigt sind durch die Gaudinischen Pässe zu gehen, ist das wirklich zu vermerken. ${ }^{24}$

21 Vgl. MiEcK, Deutschlands Westgrenze, S. $218 \mathrm{f}$.

22 Zit. nach: Metz en 1870, S. 39.

23 FIRCKS, Vertheidigung, S. 467.

24 Briefe des Generals der Infanterie von Voigts-Rhetz, S. 166. Ähnlich: TiedemanN, Erinnerungen, S. 40. Kretschman, Kriegsbriefe, S. 189. DelbrüCK, Kriegskunst, S. $397 \mathrm{f}$. SANDER, Vier Tage, S. $4 \mathrm{f}$. 
Mit der Betonung der beeindruckenden Stärke des geschlagenen Gegners wollte man zugleich die besondere eigene Leistung herausstellen. Auch wurde Marschall Bazaine entgegen aller anderslautenden Hinweise nicht als zögerlicher Feldherr, sondern als Typus eines mittelalterlichen Condottiere präsentiert. „Alles an dieser festen, gedrungenen Gestalt", so vermeldete der Korrespondent der Gartenlaube von der Gefangennahme des „Kaisers von Metz",

mit den rapiden, entschlossenen Bewegungen, mit diesem kurzgeschorenen Kopfe, mit der wie aus Eisen gegossenen Stirn, mit diesen dunklen, glühenden und verschlagenen Augen, alles das deutete auf einen Charakter, der sich mit seinem Rechte auf sich selber stellt, der den Erfolg über die Mittel setzt, und sich und seine selbstbestimmende Kraft als den Anfang und das Ende der Dinge betrachtet. ${ }^{25}$

Die Soldaten wurden zunächst in große Sammellager dirigiert und von dort schrittweise nach Deutschland abtransportiert. Wann hatte man je solche Gefangenenheere gesehen? Sedan hinzugerechnet, waren es an die 300000 Mann in weniger als zwei Monaten ${ }^{26}$. Zu Beginn des Ersten Weltkrieges hoffte man beim deutschen Vormarsch im Westen vergeblich auf derartige Erfolge. Bei Tannenberg gerieten dann immerhin knapp 100000 Russen in deutsche Gefangenschaft, in Stalingrad, im Februar 1943, 90000 Deutsche in sowjetische. Auch das waren ja ungeheure Zahlen. In den ersten Tagen nach der Metzer Kapitulation herrschten folglich chaotische Zustände:

$\mathrm{Zu} 2000$ Mann [...] kommen die armen Teufel im schauderhaftesten Regenwetter und im tiefsten Koth hier angewatet, erhalten dann Verpflegung und werden andern Tags, wenn irgend möglich, weitergeschafft [...]. Stroh zur Unterlage ist selbst für unsere eigenen Truppen, die - Gott sei Dank - in Häusern, Scheunen und Ställen untergebracht sind, nicht mehr zu haben, die armen französischen Soldaten müssen daher draußen unter ihren kleinen Leinenzelten in dem aufgeweichten Schmutz liegen. Am ersten Tag fielen auf dem Marsch schon mehrere todt nieder, nach der ersten Lagernacht holte man den folgenden Tag und heute Morgen gar 110 Todte aus dem Lager [...]. Alles schreit nach Brod. ${ }^{27}$

Schlimm traf es die 83000 Mann, die bis nach Saarlouis marschieren mußten, während ein zweites Gefangenenheer bereits ab Courcelles per Bahntransport den Weg nach Deutschland antrat ${ }^{28}$. In der deutschen Grenzstadt bot sich den Augenzeugen das Bild einer total erschöpften Armee, deren Soldaten durch Hunger, Kälte und Krankheiten tödlich geschwächt waren. Klei-

25 Gartenlaube (1870), Nr. 50, S. 843. Engels zu Bazaines Rolle wieder klar und deutlich: „Er wird unsterblich werden als der Mann, der die würdeloseste Tat in der Kriegsgeschichte Frankreichs beging, als der Mann, der 160000 Franzosen hinderte, die einschließende Armee zu durchbrechen, obwohl diese absolut schwächer war, und der sie als Kriegsgefangene auslieferte, als nichts mehr zu essen da war." Ders., Über den Krieg - XXVI, in: MEW, Bd. 17, S. 158-160, hier S. 160.

26 Insgesamt gerieten 1870/71 knapp 400000 Franzosen in deutsche Kriegsgefangenschaft. Vgl BOTZENHART, Kriegsgefangene, S. 28.

27 Brief eines preußischen Soldaten vom 2. November 1870. In: WZ (1870), Nr. 265.

2870000 Mann nahmen die Südroute über Saarbrücken: FrRCKS, Vertheidigung, S. 468. 


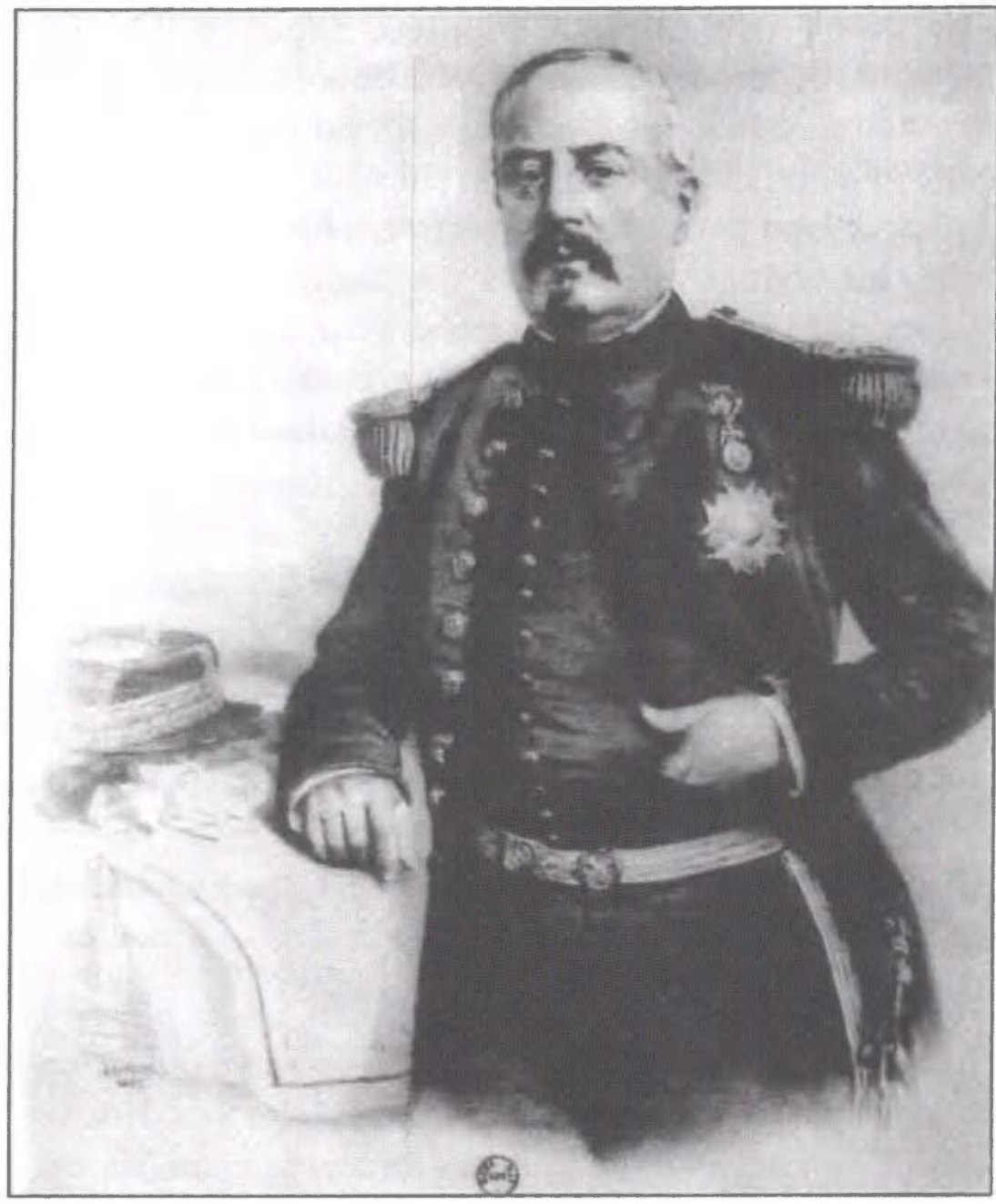

\section{Le marechal Bazaine en 1869.}

Abb. 15: Quelle: DenIs, Garnison. 
dung und Ausrüstung starrten vom gelben Lehm, in dem die Männer vor Metz gelagert hatten. In den von Ungeziefer befallenen Uniformen „steckten bleiche, abgemagerte Gestalten"29. Schwerkranke und Fiebernde, die eine längere Bahnfahrt nicht überstanden hätten, sonderte man aus und wies sie in die naheliegenden Lazarette ein. Anfang November starben dort täglich 20 bis 30 Personen, von denen die Mehrzahl der Rheinarmee zugehörig war ${ }^{30}$. Auch die Bahntransporte ins deutsche Hinterland stellten die französischen Soldaten, die zum Teil noch ihre Sommeruniformen trugen, auf eine weitere harte Probe. Häufig in offenen Wagen unterwegs, da in der Eile keine Personenwagen zu beschaffen waren, fehlten warme Kleidung und ausreichende Verpflegung. Auf der 18-stündigen Fahrt von Metz nach Berlin beispielsweise wurde nur einmal Kaffee ausgeteilt ${ }^{31}$. Schon bald boten die zu Kriegsbeginn für die Gefangeneninternierung bestimmten Festungen keinen ausreichenden Platz mehr. So ging man zur Errichtung großer, heizbarer Barackenlager über, in denen die Masse der Gefangenen aber erst nach Wintereinbruch zumutbar unterkam ${ }^{32}$. Auf dem Petersberg bei Koblenz beispielsweise wurden 10000 Gefangene aus Metz interniert. In den insgesamt 104 Holzbarakken hatten je 100 Gefangene Platz, von denen jeder einen Strohsack, ein Strohkopfpolster und zwei wollene Decken ausgehändigt bekam. Die Barakken waren mit Tischen und Bänken ausgestattet und enthielten je drei Öfen und zehn Öllampen. Ein Drahtzaun umgab das Lager ${ }^{33}$. Die Verpflegung entsprach den Vorgaben des Roten Kreuzes ${ }^{34}$. Dies galt für den einfachen Soldaten.

Offiziere dagegen konnten sich in Privatquartieren oder Hotels einmieten. Die Marschälle Bazaine, Leboeuf und Canrobert etwa wählten Kassel zu ihrem Aufenthaltsort, wo zuvor auch ihr Kaiser Wohnung genommen hatte. Die Wilhelmshöhe mag damals für ihn und seine Generale für kurze Zeit das gewesen sein, was ein halbes Jahrhundert später dem letzten deutschen Kaiser sein Dorner Exil werden sollte. In Metz selbst blieben außer den Einwohnern und denjenigen Offizieren, die ihr Ehrenwort gegeben hatten, in diesem Krieg nicht mehr gegen Deutschland zu kämpfen ${ }^{35}$, nur die Verwundeten und Schwerkranken zurück. Natürlich entwichen noch eine Reihe französischer Militärs heimlich, um sich zu den Armeen der Republik durchzuschlagen $^{36}$.

29 Sanitäts-Bericht, Bd. II, S. 173.

30 FRITSCH, Erinnerungen, S. 147-149.

31 HeIDE, Die französischen Kriegsgefangenen, S. 51.

32 Der Deutsch-Französische Krieg 1870-71, Bd. V, S. 1537 f. Dazu auch: Sanitäts-Bericht, Bd. II, S. $176 \mathrm{f}$.

33 Ibid Bd. II, S. 178.

34 HEIDE, Die französischen Kriegsgefangenen, S. 73-76.

$35 \mathrm{Vgl}$. Protokoll der Verhandlung im Anhang/Anlage VI.

36 So etwa der Hauptmann Crêmer aus Bazaines Generalstab, der sich der Regierung in Tours zur Verfügung stellte und später als General auf dem östlichen Kriegsschauplatz eine Reihe von Erfolgen erzielen konnte. 
Bei ihrem Einmarsch in die Festung bot sich den Deutschen ein Bild der Zerstörung. Bäume und Alleen waren niedergehauen, Plätze verwüstet und Straßen verbarrikadiert. Während preußische Landwehr mit wehenden Fahnen und klingendem Spiel in die Stadt einzog, wandten sich die Franzosen stumm ab. Einige Frauen zeigten sich in schwarzen Kleidern. Ein deutsches Postamt wurde noch am 29. Oktober eingerichtet ${ }^{37}$. Obwohl der provisorische Stadtkommandant Generalleutnant Kummer, um möglichen Übergriffen vorzubeugen, sogleich einen strengen $\mathrm{Erlaß}$ anschlagen ließ, blieb es fortan ruhig. Es gab sogar Stimmen, die behaupteten, Hannover habe bei der preußischen Besetzung im Jahr $1866^{38}$ einen weitaus feindseligeren Eindruck gemacht als Metz im Jahr 187039. Der traurige Anblick nötigte vielen Beobachtern Bekundungen des Mitleides ab:

Du machst Dir keinen Begriff davon, was die Franzosen gelitten haben müssen. Am Wege Massen krepierter Pferde; dann Erdhütten, d.h. aus Kot, da wohnten Soldaten drin. An den Bäumen, am Weinstock kein Blatt, das alles fraßen die Pferde. Dort steht ein Gaul [...]. Er rührt sich nicht - nun bricht er zusammen, er starb vor Hunger. Dort steht ein Maultier, angebunden an den schon toten Kollegen. Dazwischen verhungerte Gestalten, mit Schmutz bedeckt, es sind Menschen! [...] Ich ritt nach Metz, Soldaten an Krücken, Offiziere drängen sich durch die Straßen. Massen von Menschen flüchten $[\ldots]^{40}$.

Im Ruf, eine „schmutzige französische Stadt“ zu sein, stand Metz vor allem durch seine schwierige Wohnraumsituation, die weniger eine Folge des Krieges als der Rayongesetzgebung ${ }^{41}$ war, noch lange nach $1871^{42}$. Der urbanisierungshemmende Einfluß des Militärs verschärfte sich infolge der Ereignisse noch. Die neuen Herren jedenfalls rüsteten zunächst weiter auf und dachten lange nicht daran, dem zivilen Sektor Boden und Bauland preiszugeben. Unmittelbar nach dem Einmarsch kam Ärzten, Sanitätern und Pflegekräften die Aufgabe zu, den Ort und seine Umgebung gründlich zu desinfizieren sowie den französischen Kollegen bei der Betreuung der in der Stadt verbliebenen Verwundeten und Kranken zu helfen. Aus deutschen Heeresbeständen leitete man Wagenzüge mit Nahrungsmitteln in die Stadt, und sämtliche Einheiten wurden angewiesen, einen Teil ihrer Marketender zu den Gefangenen zu beordern ${ }^{43}$.

Offensichtlich wünschte die deutsche Armeeführung damals noch eine rasche Aussöhnung mit der Bevölkerung, um günstige Voraussetzungen für

37 Vgl. LiNCK, Verkehrswesen, S. 181.

38 Das Königreich Hannover hatte damals auf seiten der Österreicher gestanden.

39 Briefe des Generals der Infanterie von VOIGTS-RHETZ, S. 175.

40 KRETSCHMAN, Kriegsbriefe, S. 190. Ähnlich: VOIGTS-RHETZ, S. 175.

41 Festgelegter Raum zwischen Festungswerken und Stadt, der nicht zivil bebaut werden durfte. Nach dem neuen preußischen Rayongesetz von 1871 galt dies für einen Abstand von 1650 Metern.

$42 \mathrm{Vgl}$. WitTEnBRoCK, Stadterweiterung, S. 3.

${ }^{43}$ FIRCKS, Vertheidigung, S. $471 \mathrm{f}$. 
ihre künftige Assimilation zu schaffen. Das Denkmal Faberts blieb noch einige Zeit in schwarzen Flor gehüllt. Auf dem Turm der Kathedrale wehte sogar weiterhin die Trikolore über der Stadt ${ }^{44}$.

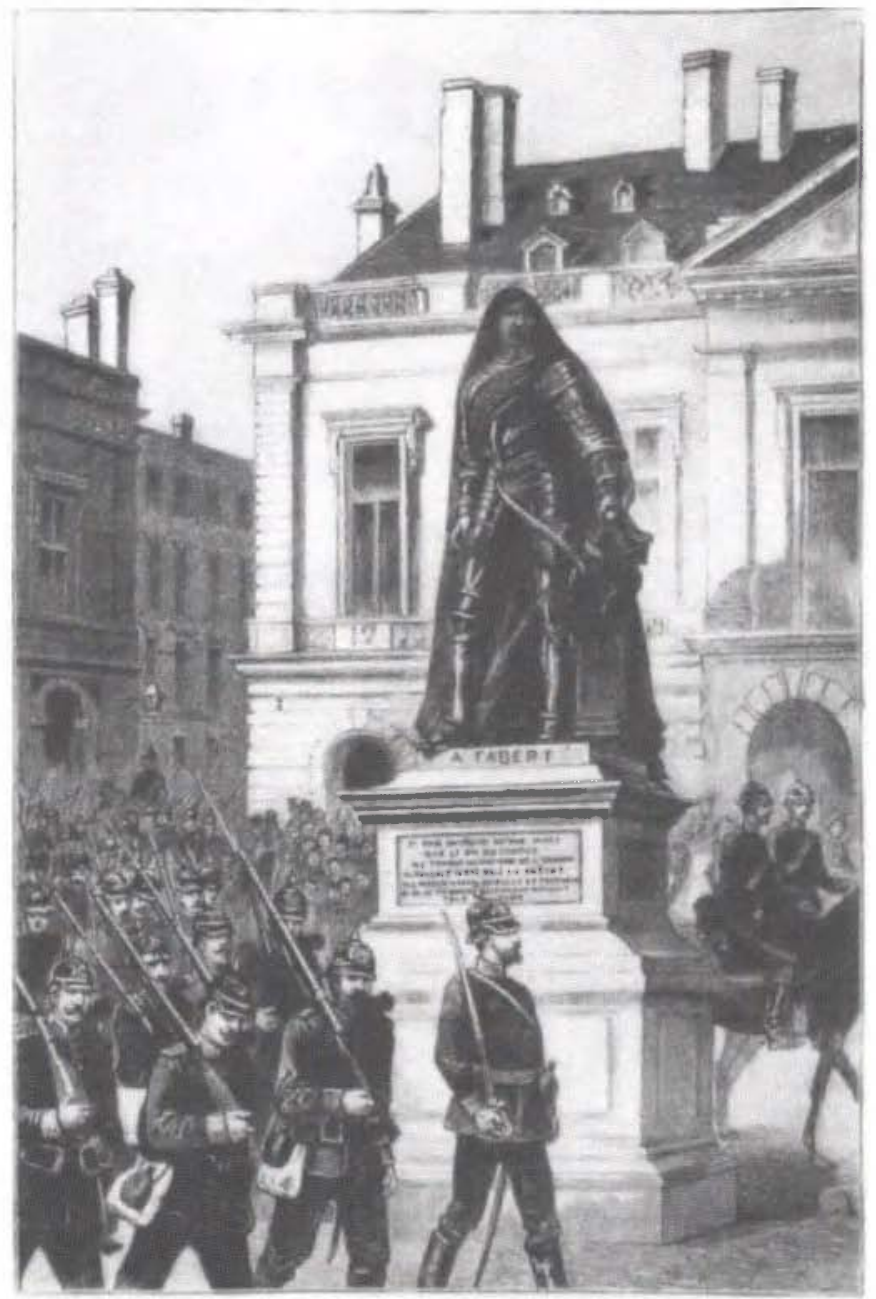

Abb. 16: Einzug der deutschen Truppen in Metz. Quelle: Fon'TANl, Wanderungen.

${ }^{44}$ FAY, Tagebuch, S. 92. Da es lebensgefährlich war, über die Turmkuppeln zur Fahnenstange zu gelangen, war das französische Hoheitszeichen auch nach der Kapitulation hängen geblieben. Später wurden auf das Abhängen 100 Taler ausgesetzt. Erst am 16. Juli 1874 gelang es einem Brandenburger Pionier, die französische Flagge einzuholen und durch eine riesige schwarz-weiß-rote Fahne zu ersetzen. Ri.GENSBI:RG, 1870/ 71, Bd. 2, S. 257. 